GANIT J. Bangladesh Math. Soc. (ISSN 1606-3694) 39 (2019) 127-140

DOI: https://doi.org/10.3329/ganit.v39i0.44168

\title{
A COMPARATIVE ANALYSIS OF THE BLACK-SCHOLES- MERTON MODEL AND THE HESTON STOCHASTIC VOLATILITY MODEL
}

\author{
Tahmid Tamrin Suki ${ }^{*}$ and A B M Shahadat Hossain \\ Department of Applied Mathematics, University of Dhaka, Dhaka-1000, Bangladesh \\ *Correspondence Author, e-mail: tts.suki24@gmail.com
}

Received: 06-07-2019

Accepted: 11-11-2019

\begin{abstract}
This paper compares the performance of two different option pricing models, namely, the BlackScholes-Merton (B-S-M) model and the Heston Stochastic Volatility (H-S-V) model. It is known that the most popular B-S-M Model makes the assumption that volatility of an asset is constant while the H-S-V model considers it to be random. We examine the behavior of both B-S-M and H$\mathrm{S}-\mathrm{V}$ formulae with the change of different affecting factors by graphical representations and hence assimilate them. We also compare the behavior of some of the Greeks computed by both of these models with changing stock prices and hence constitute 3D plots of these Greeks. All the numerical computations and graphical illustrations are generated by a powerful Computer Algebra System (CAS), MATLAB.
\end{abstract}

Keywords: Stochastic volatility, Heston model, Black-Scholes-Merton model, Skewness and kurtosis, Affecting factors, Greeks.

\section{Introduction}

Fischer Black, Myron Scholes and Robert Merton proposed the B-S-M Model in 1973 which was the first ever analytic option pricing model [1]. Afterwards this model has been treated as the benchmark model in the study of Mathematical Finance. In 1997, Merton and Scholes received 'The Nobel Memorial Prize in Economic Science' for their novel invention. It is broadly used to calculate the fair value of European options. This model is the foundation of most of the option pricing models. However it sometimes provides inexact prices when tested against real market data. The reason behind this is B-S-M model is generated on the basis of some inflexible assumptions which are hardly accepted in real market. For example, the assumption of constant volatility is a great disparity in the market [2].

Many researchers contributed a lot in the evolution of the stochastic volatility option pricing models. Among them Johnson and Shanno [3], Wiggins [4], Hull and White [5] played massive important role. To address volatility related mispricing, the $\mathrm{H}-\mathrm{S}-\mathrm{V}$ model has been proposed in

(C) GANIT: Journal of Bangladesh Mathematical Society, 2019 
literature as a generalization of B-S-M model in 1993 [6]. This model considers the randomness of volatility of the underlying asset and derives a closed form solution for pricing European call (or put) options when the underlying asset price follows non-Gaussian distribution [4], [7], [8]. The $\mathrm{H}-\mathrm{S}-\mathrm{V}$ model allows sufficient flexibility of the parameters for a better fit than that obtained by the B-S-M model. We investigate the relative pricing by a cross-checking with real market data. Hence the objective of this paper is to investigate the variation of the option prices and value of some Greeks calculated under different circumstances for both of these models and hence compare them. Here we comment on different Suki and Hossain situations when these models perform similarly or dissimilarly with two different data sets.

The B-S-M model considers stock price follows a geometric Brownian motion and derivatives exchanged on the stock have fair prices under risk-neutral valuation [1] , [5]. It is the grounds of modern option pricing theory. To derive the theoretical option prices it uses five key determinants or affecting factors: stock price $(S)$, strike price $(K)$, volatility $(\sigma)$, time to expiration $(T)$ and shortterm (risk free) interest rate $(r)$. The B-S-M model assumes volatility to be a constant [2] while the $\mathrm{H}-\mathrm{S}-\mathrm{V}$ model considers the fact that volatility of the stock price is itself random and follows a CIR process [9]. The second assumption is more compatible with the real market.

This paper purely provides comparative analysis of the results obtained by the models under discussion. We compare the change in option prices calculated by both B-S-M and H-S-V models with the variation of some affecting factors. Also the comparison for different Greeks under both models is added for two different data sets. For more details the readers are referred to go through [4], [5], [6], [13], [14], [16] etc.

\section{Our Models}

In this section, we discuss both the B-S-M and H-S-V option pricing models in brief. We refer the readers to see [1], [2], [6] to know more about these models.

\subsection{The B-S-M Option Pricing Formula}

The dynamics of a risky asset, such as stock, under the B-S-M model can be characterized by the following Stochastic Differential equation (SDE) [1],

$$
\begin{aligned}
& d S_{t}=r S_{t} d t+\sigma_{b s m} S_{t} d B_{t}, \forall t \in[0, T] \\
& S_{t}=S_{0} \text { when } t=0 .
\end{aligned}
$$

Where $B=\left(B_{t}: 0 \leq t \leq T\right)$ is a Wiener Process under the given probability measure of the riskneutral world, $P$; the parameter $r$ is the risk-free interest rate and $\sigma_{b s m}$ denotes the volatility of the risky asset.

The Partial Differential Equation (PDE) provided by this model for any underlying derivative $C$ and current stock price $S$ is, 


$$
\frac{\partial C}{\partial t}+r S \frac{\partial C}{\partial S}+\frac{1}{2} \sigma_{b s m}^{2} S^{2} \frac{\partial^{2} C}{\partial S^{2}}=r C
$$

Then a European call option price admits the equation (2) with the following Boundary Conditions (BCs),

$$
\left\{\begin{array}{l}
C(S, T)=\max (S-K, 0) \\
C(0, t)=0 \\
C(S \mapsto \infty, t)=S-K e^{\wedge}(-r(T-t))
\end{array}\right.
$$

The a European call option price at time $t$ with exercise price $K$, maturity $T$, constant interest- rate $r$ and volatility $\sigma_{b s m}$ under this model is,

$$
C=S N\left(d_{+}\right)-K e^{-r \tau} N\left(d_{-}\right)
$$

Where $\quad d_{ \pm}=\frac{\ln (S / K)+\left(r \pm 1 / 2 \sigma_{b s m}^{2}\right) \tau}{\sigma_{b s m} \sqrt{\tau}} \quad \tau=T-t$.

Here $N(z)$ is the standard normal cumulative distribution function.

\subsection{The H-S-V Option Pricing Formula}

On the other hand, the dynamics of the risky asset in H-S-V model is derived from the following SDEs [6], [10]:

$$
d S_{t}=r S_{t} d t+\sqrt{v_{t}} S_{t} d B_{t}^{S}
$$

where $v_{t}$, the instantaneous variance follows a CIR process as follows,

$$
d v_{t}=\kappa\left(\theta-v_{t}\right) d t+\sigma \sqrt{v}_{t} d B_{t}^{v}
$$

Here $B_{t}^{S}$ and $B_{t}^{v}$ are Wiener Processes with correlation $\varrho$ or equivalently, with covariance $\varrho d t$ i.e.

$$
d B_{t}^{S} d B_{t}^{v}=\varrho d t
$$

The parameters in the above equations represent the followings:

(i) $\quad r=$ the rate of return of the asset.

(ii) $\theta=$ the long variance or the expected value of $v_{t}$ which tends to $\theta$.

(iii) $\kappa=$ the rate at which $v_{t}$ reverts to $\theta$.

(iv) $\sigma=$ the volatility of the volatility which calculates the variance of $v_{t}$.

If the parameters follow the Feller condition as given below, then the drift is large enough for the variance $v_{t}$ to be firmly positive.

$$
2 \kappa \theta>\sigma^{2}
$$


According to Cox, Ingersoll and Ross (1985) the mean and variance of $v_{t}$ conditional on the value of $v_{s}(t>s)$ are respectively [9],

$$
\left.\begin{array}{c}
m=\mathbb{E}\left[v_{t} \mid v_{s}\right]=\theta+\left(v_{s}-\theta\right) e^{-\kappa(t-s)} \\
\left.v_{s}\right]=\frac{v_{s} \sigma^{2} e^{-\kappa(t-s)}}{\kappa}\left(1-e^{-\kappa(t-s)}\right)+\frac{\theta \sigma^{2}}{2 \kappa}\left(1-e^{-\kappa(t-s)}\right)^{2}
\end{array}\right\}
$$

The effect of the mean reversion rate $\kappa$ on the moments is intuitive. When $\kappa \mapsto \infty$ the mean $m$ approaches the mean reversion rate $\theta$ and the variance var approaches 0 . As $\kappa \mapsto 0$ then $m \mapsto v_{s}$ i.e. the current level of variance var $\mapsto \sigma^{2} v_{t}(t-s)$. Here all the parameters $\kappa, \theta, \sigma, \varrho$ are time and state homogeneous. In other words, Heston model assumes that the variance is a stochastic process rather than a constant that,

- exhibits a proneness to revert towards a long term mean $\theta$ at the rate $\kappa$

- displays a volatility proportional to the square root of its level

- and whose source of stochasticity is correlated (with correlation $\varrho$ ) with the randomness of the underlying assets's price process.

Now the PDE under H-S-V model for the underlying derivative $C$ is [5]

$$
\frac{\partial C}{\partial t}+\frac{1}{2} v S^{2} \frac{\partial^{2} C}{\partial S^{2}}+\tilde{n} \sigma S v \frac{\partial^{2} C}{\partial S \partial v}+\frac{1}{2} \sigma^{2} v \frac{\partial^{2} C}{\partial v^{2}}+r S \frac{\partial C}{\partial S}-r C+\{\kappa(\theta-v)-\lambda v\} \frac{\partial C}{\partial v}=0
$$

Here $\lambda$ represents the market price of volatility risk. For a European call option, the above PDE (10) satisfies the following BCs:

$$
\left\{\begin{array}{c}
C(S, v, T)=\max (S-K, 0) \\
C(0, v, t)=0 \\
\frac{\partial C}{\partial S}(S \mapsto \infty, v, t)=1 \\
\frac{\partial C}{\partial t}(S, 0, t)+r S \frac{\partial C}{\partial S}(S, 0, t)+\kappa \theta \frac{\partial C}{\partial v}(S, 0, t)-r C(S, 0, t)=0 \\
C(S, v \mapsto \infty, t)=S
\end{array}\right.
$$

Taking $A=r S \frac{\partial}{\partial S}+\frac{1}{2} v S^{2} \frac{\partial^{2}}{\partial S^{2}}+\tilde{n} \sigma S v \frac{\partial^{2}}{\partial S \partial v}+\frac{1}{2} \sigma^{2} v \frac{\partial^{2}}{\partial v^{2}}+\{\kappa(\theta-v)-\lambda v\}$, the Heston PDE can be rewritten as $\frac{\partial C}{\partial t}+A C-r C=0$. ' $A$ ' is stated as the generator of the H-S-V model and the first two terms of $A$ as the generator of B-S-M with $v=\sqrt{\sigma_{b s m}}$. The value of a European call option under H-S-V model is,

$$
C(K)=S \psi_{1}-K e^{-r \tau} \psi_{2}
$$


Where $\psi$ is the CDF followed by the stock price return in $\mathrm{H}-\mathrm{S}-\mathrm{V}$ model defined by [1] ,

$$
\psi_{j}=\operatorname{Pr}\left(\ln S_{t}>\ln K\right)=\frac{1}{2}+\frac{1}{\pi} \int_{0}^{\infty} \operatorname{Re}\left[\frac{e^{-i \phi \ln K} f_{j}(\phi ; x, v)}{i \phi}\right] d \phi
$$

with the characteristic functions $f_{j}$, such that,

$$
f_{j}(\phi ; x, v)=\mathbb{E}\left[e^{i \phi x_{T}}\right]=\exp \left(C_{j}(\tau, \phi)+D_{j}(\tau, \phi) v+i \phi x\right)
$$

Here we have,

$$
\left\{\begin{array}{c}
g_{j}=\frac{b_{j}-\tilde{n} \sigma i \phi+d_{j}}{b_{j}-\tilde{n} \sigma i \phi-d_{j}} \\
d_{j}=\sqrt{\left(\tilde{n} \sigma i \phi-b_{j}\right)^{2}-\sigma^{2}\left(2 u_{j} i \phi-\phi^{2}\right)} \\
C_{j}(\tau, \phi)=r \phi i \tau+\frac{a}{\sigma^{2}}\left[\left(b_{j}-\tilde{n} \sigma i \phi+d_{j}\right) \tau-2 \ln \left(\frac{1-g_{j} e^{d_{j} \tau}}{1-g_{j}}\right)\right] \\
D_{j}(\tau, \phi)=\frac{b_{j}-\tilde{n} \sigma i \phi+d_{j}}{\sigma^{2}}\left(\frac{1-e^{d_{j} \tau}}{1-g_{j} e^{d_{j} \tau}}\right)
\end{array}\right.
$$

$\forall j=1,2$ with $x=\ln S, \tau=(T-t), u_{1}=\frac{1}{2}, u_{2}=-\frac{1}{2}, b_{1}=\kappa+\lambda-\tilde{n} \theta, b_{2}=\kappa+\lambda, a=\kappa \theta$ and $i=\sqrt{-1}$.

\subsubsection{Effect of Heston Parameters}

As we already mentioned that, the $\log$ stock price i.e. $\ln S_{t}$ under Heston model follows nonGaussian distribution [7]. Depending on the choice of parameters, $\ln S_{T}$ is able to display skewness and excess kurtosis at maturity. Here we study the effect of correlation $\varrho$ and volatility of variance $\sigma$ on this $\log$ stock value distribution. For this we will use the same parameter settings from the first Table of [1] for a 6 months option.

Table 1: Default Parameters Provided by Steven L. Heston

\begin{tabular}{|c|c|c|c|c|c|c|c|c|}
\hline$S_{0}$ & $K$ & $r$ & $v_{0}$ & $\kappa$ & $\theta$ & $\lambda$ & $\sigma$ & $\varrho$ \\
\hline 100 & 100 & 0 & 0.01 & 2 & 0.01 & 0 & 0.1 & 0 \\
\hline
\end{tabular}

Effect of Correlation, $\varrho$

Since $\varrho$ is the correlation between the stock price process and the volatility process, it controls the heaviness of tails i.e. the skewness of the density of $\ln S_{T}$. If $\varrho=0$ the skewness will be close to zero. The following Figure 1 drawn using Table 1 can give us a better idea of the effect of $\varrho=-0.8, \varrho=0$ and $\varrho=0.8$ respectively on the skewness of the Heston Density Function. 


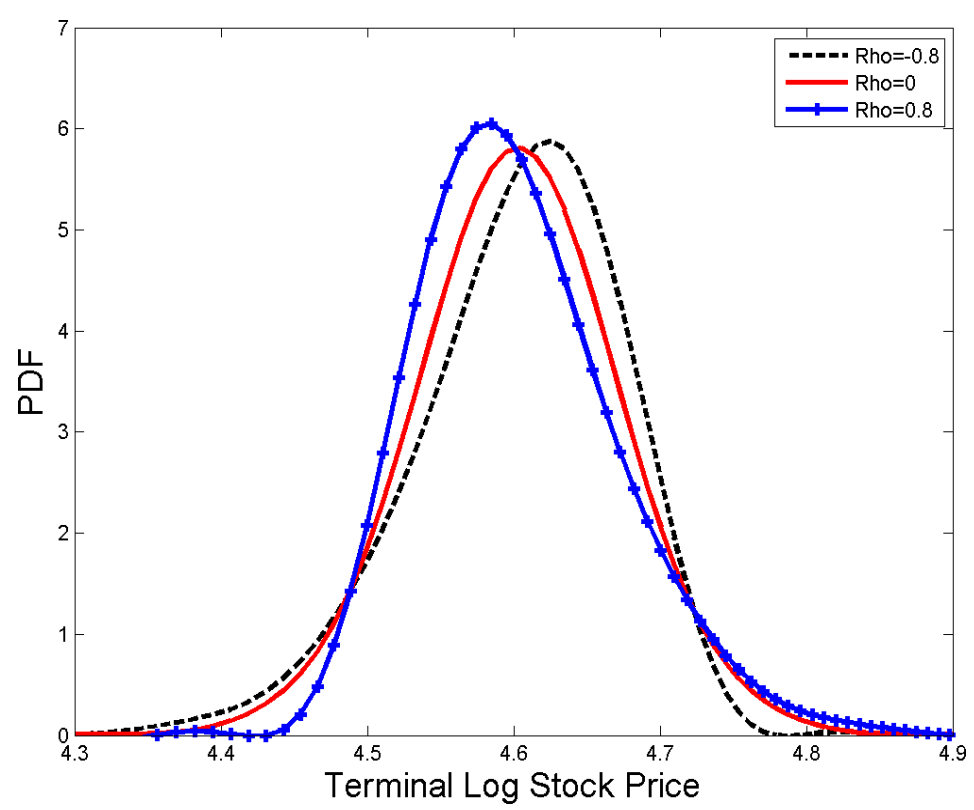

Figure 1: The effect of Correlation on Heston Density Function.

We observe from Figure 1 that, the probability densities are positively skewed for $\varrho>0$ that is the variance increases with a rise in stock price. This widens the right tail of the distribution and squeezes the left tail. In case of Out-The-Money (OTM) calls i.e. calls for which $S_{t}<K$, the strike price lies in the right tail and hence deep OTM calls obtained by H-S-V model should be more costly than those by the B-S-M model. On the other hand, In-The-Money (ITM) call options for which $S_{t}>K$, contains a strike price lying in the left tail. In such cases, deep ITM calls from the volatility model should be cheaper than those obtained by B-S-M for $\varrho>0$ due to less weight assigned to the left tail. Again the probability densities are negatively skewed when $\varrho<0$ which means the volatility increases if there is a decrease in stock price. This results into a fattening of left tail and a thinning of the right tail of the distribution. Some counter behavior is observed for $\varrho<0$ in case of both ITM and OTM call options. That means the Heston model should produce more expensive deep ITM calls from than those generated by the B-S-M model. Thus, deep OTM calls by $\mathrm{H}-\mathrm{S}-\mathrm{V}$ model should be less expensive.

\section{Effect of Volatility of Variance, $\sigma$}

The volatility of variance, $\sigma$ effects the peak i.e. the kurtosis of the distribution of the $\log$ stock price under $\mathrm{H}-\mathrm{S}-\mathrm{V}$ model. When $\sigma \approx 0$ the $\log$ stock price is normally distributed since the randomness of the variance process $v_{t}$ as shown in equation (6) drops out. The effect of $\sigma$ can be illustrated in the following Figure 2 for Table 1 and $\sigma \approx 0(\sigma=0.0001), \sigma=0.1, \sigma=0.2$ respectively. It is clear that, increasing $\sigma$ increases the peaks of the distribution and create heavy tails on both sides. 


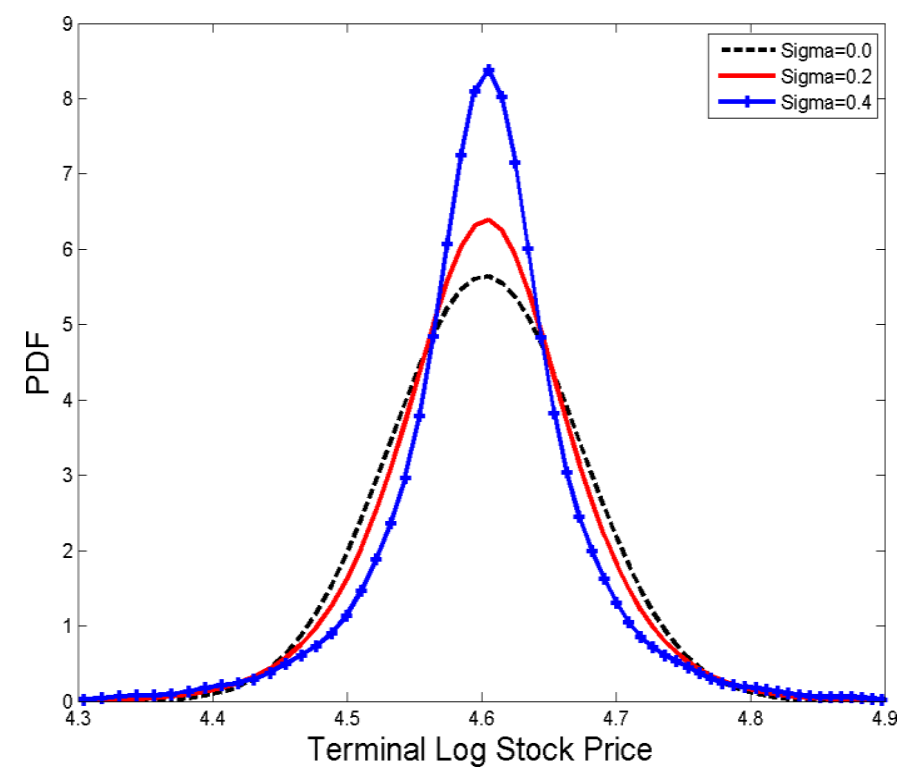

Fig. 2: The effect of Volatility of Variance on Heston Density Function.

\section{Comparison between B-S-M and H-S-V Model}

If we substitute $\sigma=0$ and $\theta=v_{0}$ into equation (10) along with $\lambda=0$, it will produce B-S-M PDE from the one provided by H-S-V model with $\sigma_{b s m}=\sqrt{v_{0}}$ [2]. Also if we set $\sigma=0$ then the Brownian Motion component in equation (6) vanishes which results into $\operatorname{Var}\left[v_{t} \mid v_{0}\right]=0$ from equation (9). This produces time-varying deterministic probability. Again, setting $\theta=v_{0}$ we get from (9), $\mathbb{E}\left[v_{t} \mid v_{0}\right]=v_{0}$ which is clearly constant. As a consequence the Heston price under these parameter values will be similar to the B-S-M price. Hence we can say that, B-S-M model is a special case of H-S-V model. But we cannot simply substitute $\sigma=0$ directly into the pricing functions. Otherwise it will cause invalid terms produced due to division by zero in $C_{j}(\tau, \phi)$ and $D_{j}(\tau, \phi)$ in equations (15). In this work, we consider, $\sigma \approx 0$ say $\sigma=0.0001$. For computations we use two different data sets as given below. The first data set is collected from [9] and second one is from [15].

\section{Data Set 1:}

$$
\left\{S_{0}=100, K=100, r=0.05, v_{0}=0.07, \kappa=0, \theta=0.07, \lambda=0, \sigma=0.0001, \tilde{n}=-0.8, T=0.5\right\}
$$

\section{Data Set 2:}

$$
\left\{S_{0}=100, K=100, r=0.00135, v_{0}=0.028087, \kappa=2.9315, \theta=0.01, \lambda=0, \sigma=0.1, \tilde{n}=0.5, T=1\right\}
$$

Apparently, the first data set satisfies $\theta=v_{0}$ and $\sigma \approx 0$ but the second one does not.

For these different data sets, we observe the variations in call option prices obtained from both the $\mathrm{H}-\mathrm{S}-\mathrm{V}$ and B-S-M models with the changing affecting factors as well as compare the Greeks 
calculated under these models with varying stock prices. We simply assume, $v=\sqrt{\sigma_{b s m}}$ i.e. $\sigma_{b s m}=v^{2}$.

\subsection{Comparison for Affecting Factors}

There are six factors affecting the stock option price under the B-S-M model [1]. They are namely, the current stock price, strike price, time to maturity, risk-free interest rate, volatility of the stock price etc. as we mentioned earlier. These factors play vital role in the life of an option. We will discuss the behavior of our models for changes in some of the affecting factors mentioned above.

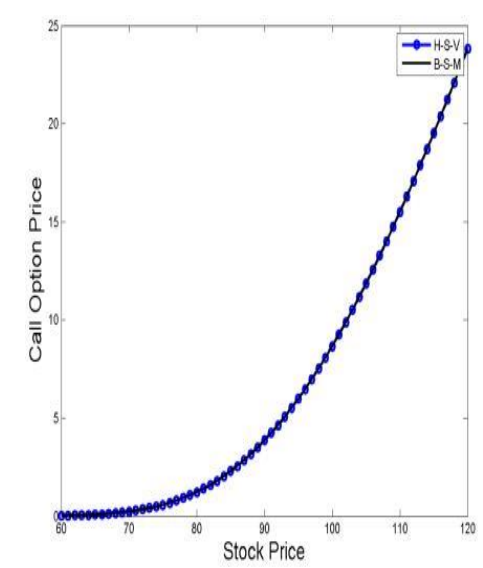

(a)

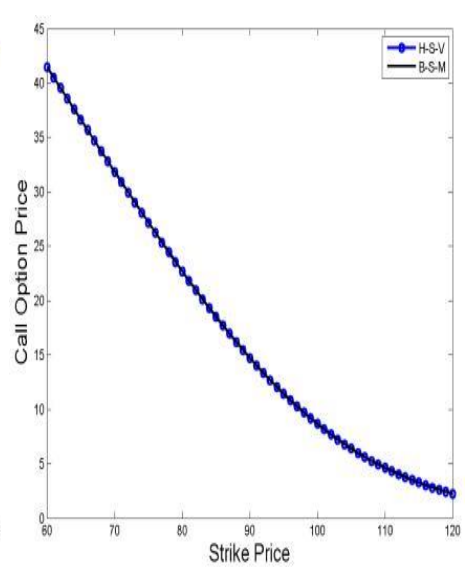

(b)

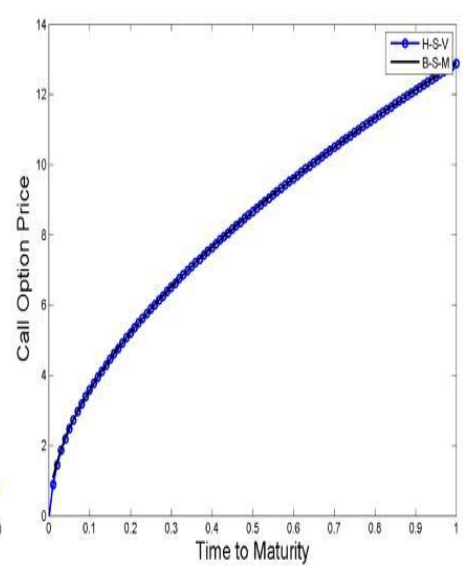

(c)

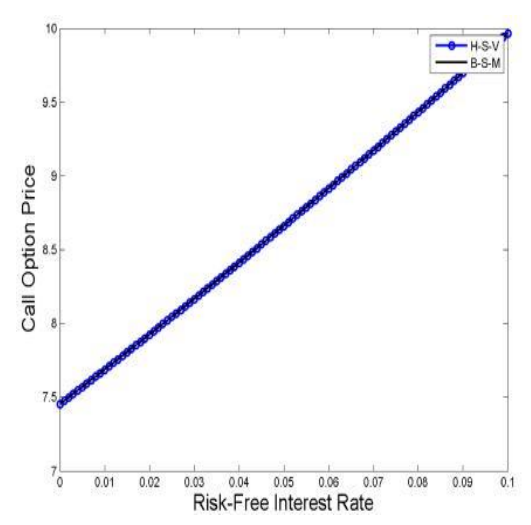

(d)

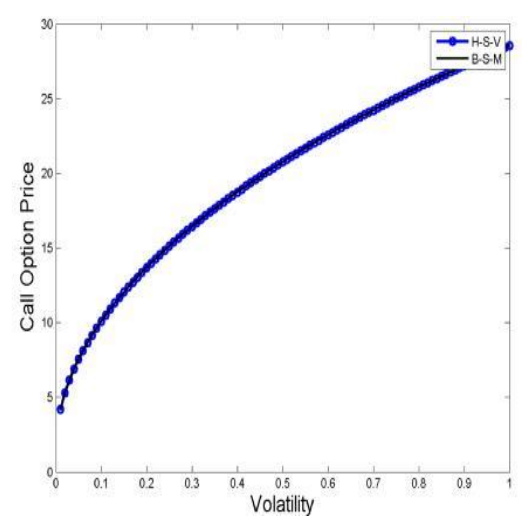

(e)

Fig. 3: Comparison for affecting factors using Data Set 1.

In Figure 3, clearly for the parameters mentioned in Data Set 1, we get identical call option values for both models no matter whatever the varying affecting factor is. That means changing any factor effects these models in similar way and gives uniform call values. But this is not the case for the second data set. 


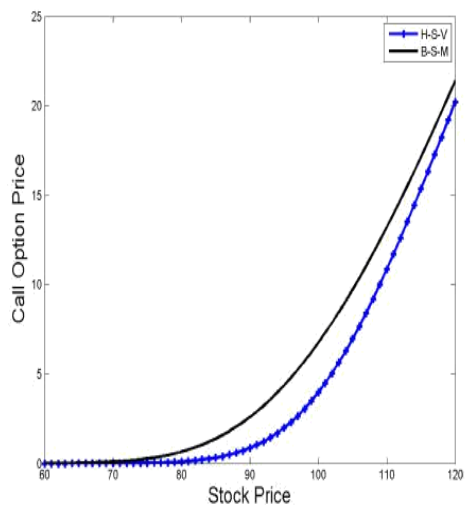

(a)

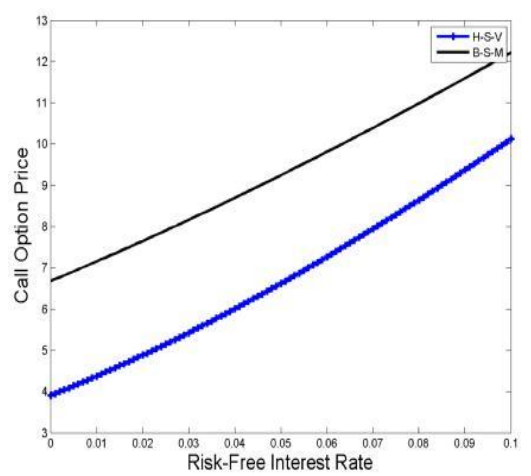

(d)

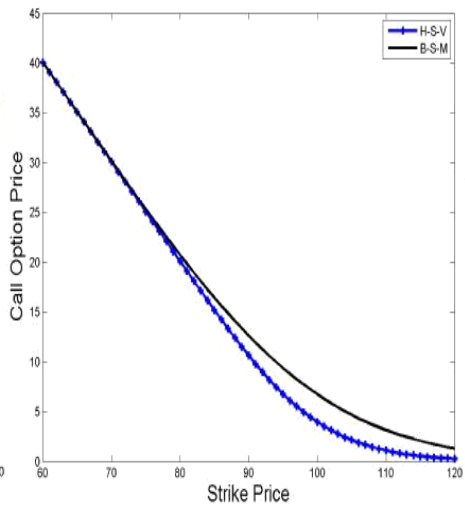

(b)

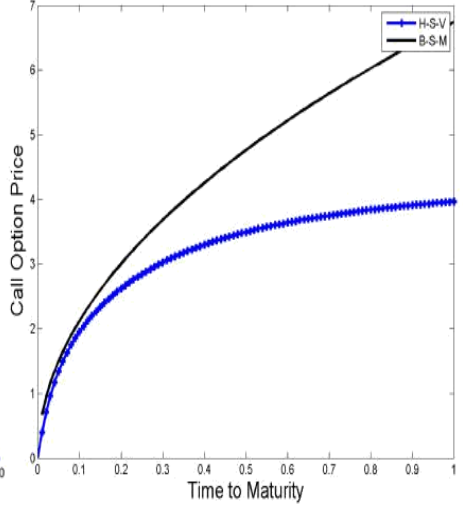

(c)

Figure 4: Comparison for affecting factors using Data Set 2.

Now for Data Set 2, Figure 4(a) shows that the call option values obtained by H-S-V model are less than those of B-S-M model except for some initial stocks. But both these model behave in quite a likely way as we keep changing the stock prices and result into two increasing curves. Similarly, for the change in other affecting factors the call values dynamic obtained by the models under discussion not really coincide but act equivalently. In each case, Heston call values are less than B-S$\mathrm{M}$ prices (apart from some primary values). For both cases, the blue curves denote variation of option prices by H-S-V model whereas the black ones denote the same for B-S-M model.

\subsection{Comparison for Greeks}

We know that, the quantities which represent the sensitivity of the price of different derivatives (e.g. options) to a change in underlying parameters are known as Greeks. The value of a portfolio of financial instruments depends. Collectively these Greeks are also called the risk sensitivities, risk measures or hedge parameters. We will discuss the variation in some of the European call option Greeks for both of our models. Observing the following Table 2, we are certain that the formulae for calculating Greeks under $\mathrm{H}-\mathrm{S}-\mathrm{V}$ model are much more complex in structure than the Greeks provided by B-S-M model (see [1], [9]). 
Table 2: Greeks in B-S-M and H-S-V models for European Call Options

\begin{tabular}{|l|l|l|}
\hline $\begin{array}{c}\text { Definitions of Greek } \\
\text { Letters }\end{array}$ & \multicolumn{1}{|c|}{$\begin{array}{c}\text { Formulae for H-S-V } \\
\text { Model }\end{array}$} & $\begin{array}{c}\text { Formulae for B-S-M } \\
\text { Model }\end{array}$ \\
\hline$\Delta=\frac{\partial C}{\partial S}$ & $\frac{1}{2}+\frac{1}{\pi} \int_{0}^{\infty} \operatorname{Re}\left[\frac{e^{-i \phi \ln K} f_{1}}{i \phi}\right] d \phi$ & $N\left(d_{1}\right)$ \\
\hline$\Gamma=\frac{\partial^{2} C}{\partial S^{2}}=\frac{\partial \Delta}{\partial S}$ & $\frac{1}{\pi S_{0}} \int_{0}^{\infty} \operatorname{Re}\left[e^{-i \phi \ln K} f_{1}\right] d \phi$ & $\frac{N^{\prime}\left(d_{1}\right)}{S_{0} \sigma_{b s m} \sqrt{\tau}}$ \\
\hline$\rho=\frac{\partial C}{\partial \tau}$ & $K \tau e^{-r \tau}\left[\frac{1}{2}+\frac{1}{\pi} \int_{0}^{\infty} \operatorname{Re}\left[\frac{e^{-i \phi \ln K} f_{2}}{i \phi}\right] d \phi\right]$ & $K e^{-r \tau} N\left(d_{2}\right)$ \\
\hline$\vartheta=\frac{\partial C}{\partial v}$ & $2 \sqrt{v_{0}}\left[\frac{S_{0}}{\pi} \int_{0}^{\infty} \operatorname{Re}\left[\frac{e^{-i \phi \ln K} f_{1} D_{1}}{i \phi}\right] d \phi\right]$ & $S_{0} \sqrt{\tau} N^{\prime}\left(d_{1}\right)$ \\
\hline & $-2 \sqrt{v_{0}}\left[\frac{K e^{-r \tau}}{\pi} \int_{0}^{\infty} \operatorname{Re}\left[\frac{e^{-i \phi \ln K} f_{2} D_{2}}{i \phi}\right] d \phi\right]$ & \\
\hline
\end{tabular}

Using the above table, we compute different Greeks under Heston model as well as Black Scholes model. Hence we examine the behavior of these Greeks with the change in stock price.

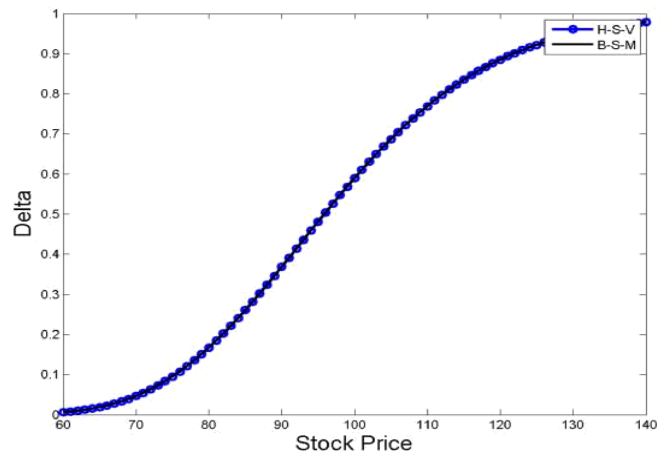

(a)

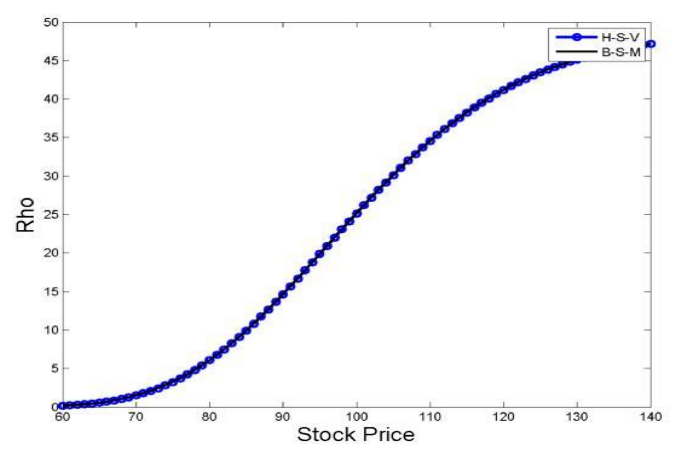

(c)

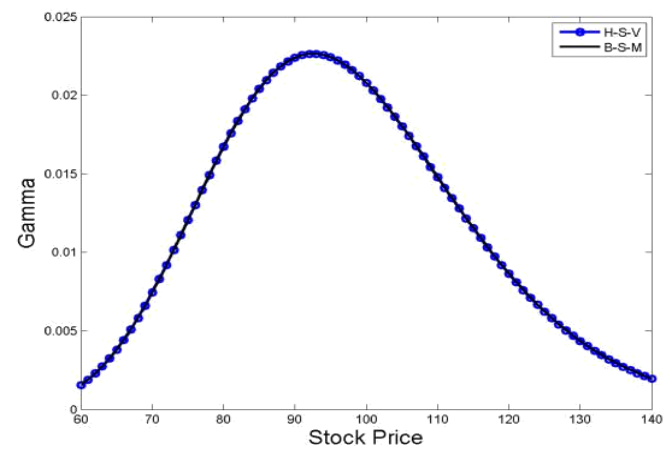

(b)

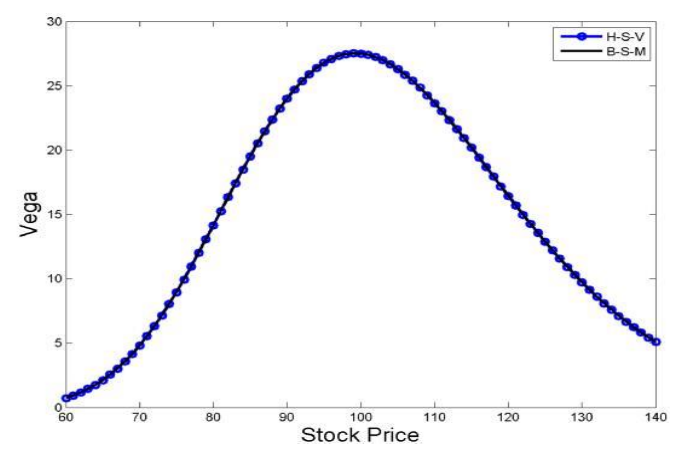

(d)

Fig. 5: Comparison for Greeks using Data Set 1. 
Using the parameter setting from Data Set 1, uniform Greek values at each stock value and hence overlapping configurations are captured for both the models.

Let us consider Data Set 2 for calculating different Greeks. The following Figure 6(a) displays that the Delta calculated from the H-S-V model is larger than the Delta given by B-S-M equation for ITM options, while the Delta in the Heston model is comparatively smaller for OTM options. This result may be fruitful for delta hedging when an investor considers about his/her portfolio's risk.

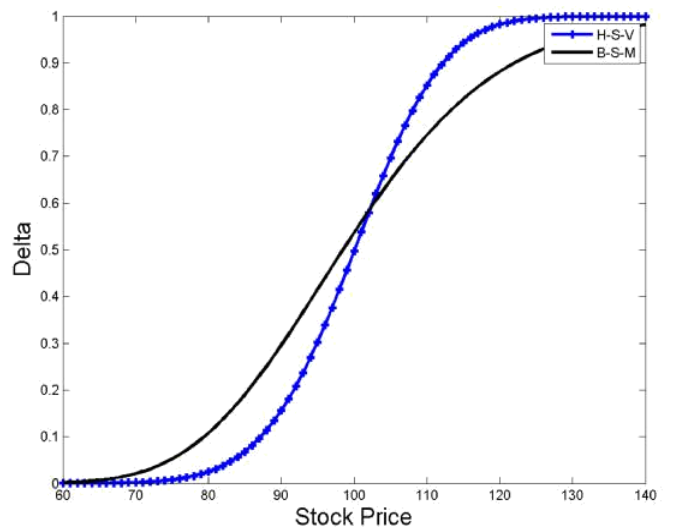

(a)

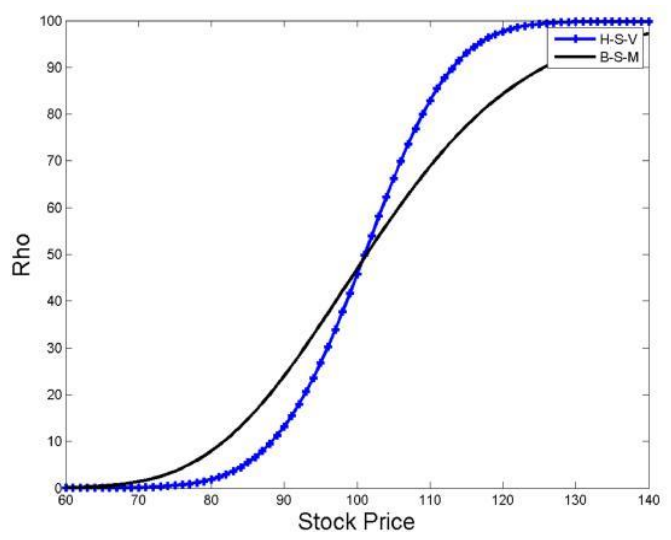

(c)

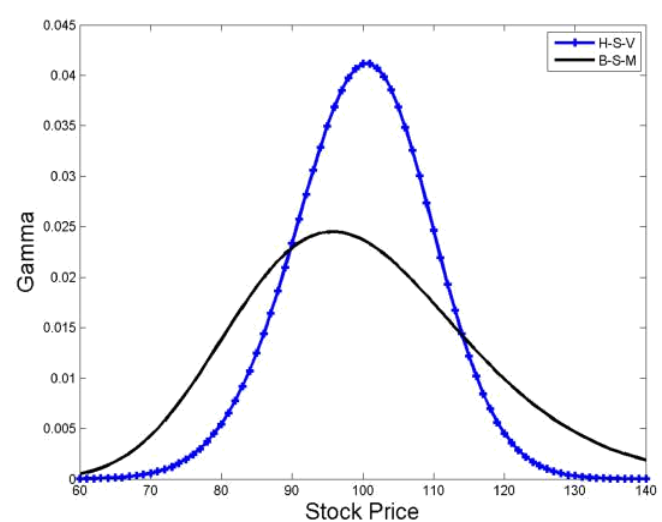

(b)

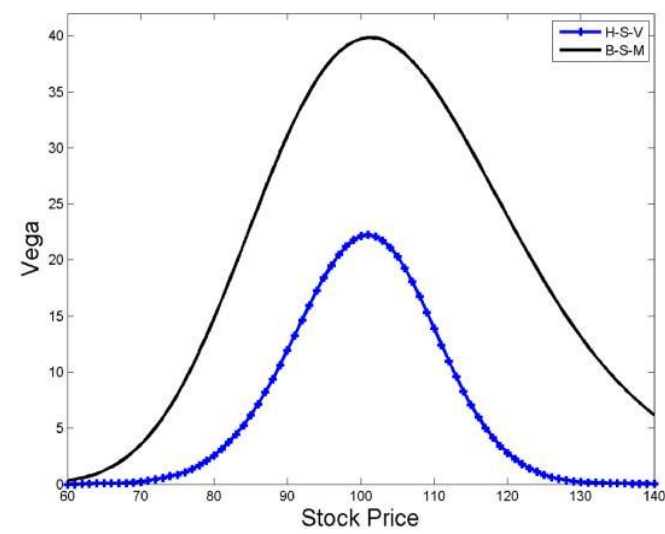

(d)

Fig. 6: Comparison for Greeks using Data Set 2.

Figure 6(b) illustrates that the Gammas computed for different stock prices by both of our models fluctuate a lot from each other but still they display similar type of behavior. Thus the values of Deltas for both these models ripple a lot from each other for different stock prices for Data Set 2. The Rho from our models for this data set display quite same type of behavior as Heston Delta with the alternating stock prices. Hence the graphs result in alike shapes in Figure 6(c). Now the Vega calculated using the formula for $\mathrm{H}-\mathrm{S}-\mathrm{V}$ model given in Table 2 has lower peak at maturity than the one computed by B-S-M model. That means, for this particular data set B-S-M model has larger values of Vega than those of H-S-V model. 


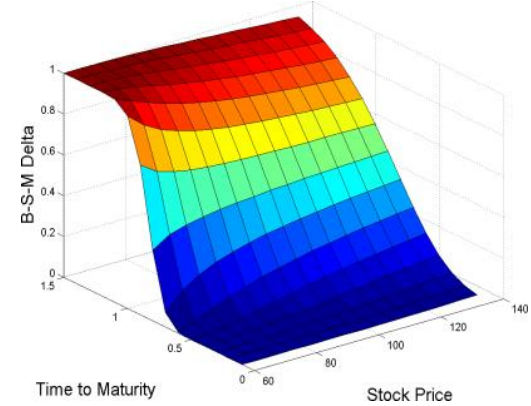

(a)

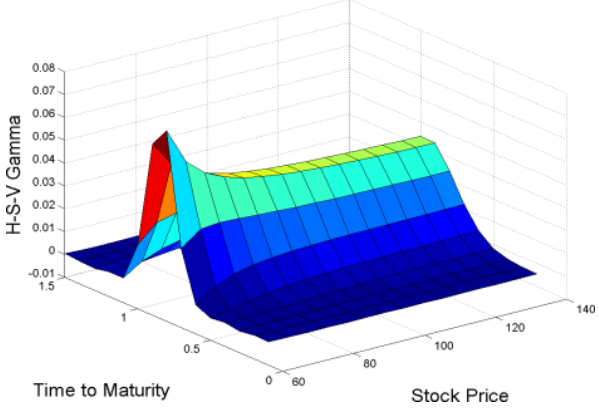

(c)

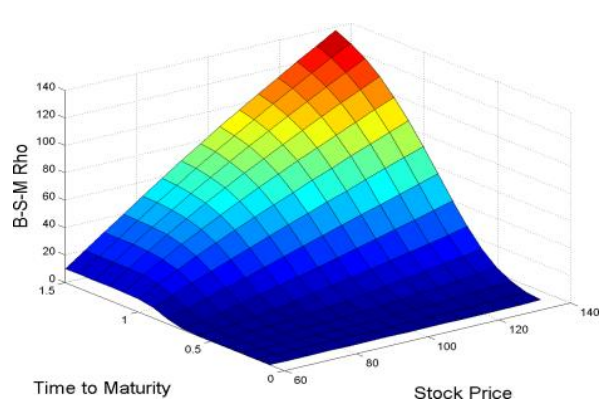

(e)

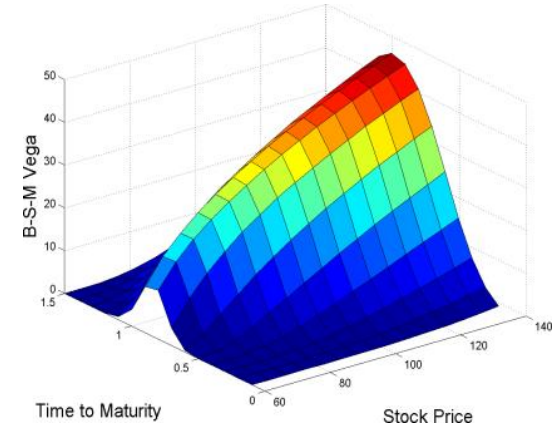

(g)

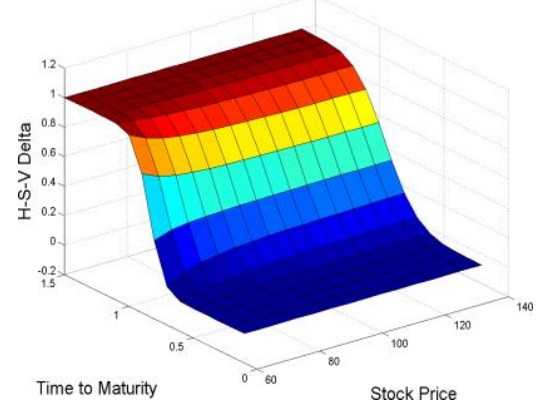

(b)

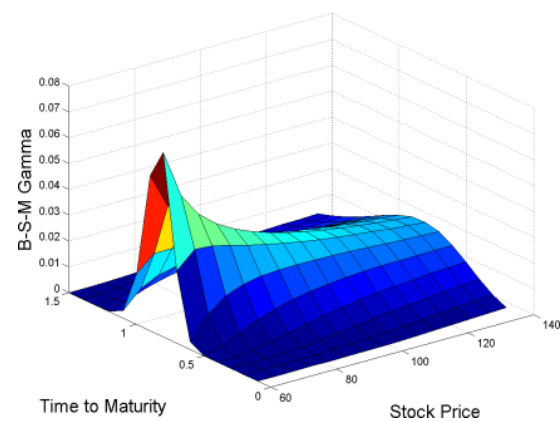

(d)

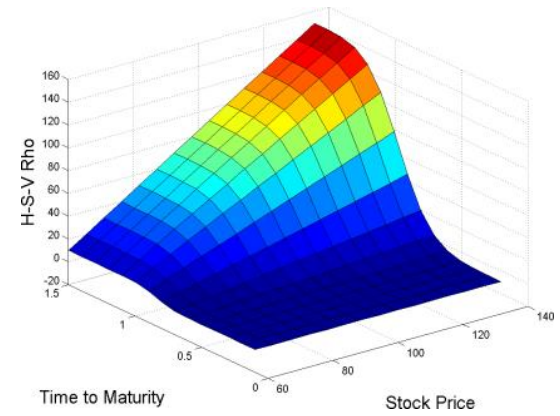

(f)

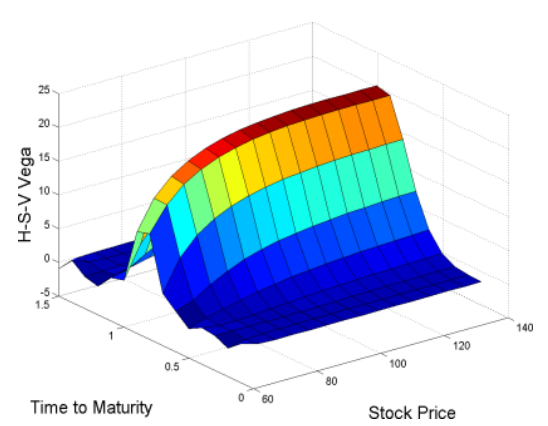

(h)

Fig 7: Greeks (3D) for B-S-M and H-S-V Models respectively. 
Evidently, if we draw 3D plots for all these Greeks calculated under both B-S-M and H-S-V models will be identical with parameters in Data Set 1. But the following Figures 7 show the differences between these Greek 3D Plots drawn for both these models with parameters selected from Data Set 2. All the 3D diagrams in the first column display B-S-M Greeks while those in the second column are for Greeks under H-S-V model.

\section{Conclusion}

In this paper, we essentially observe that, with the settings $\theta=v_{0}$ (i.e. initial volatility=long run average price variance) and $\sigma \approx 0$ (followed by Data Set 1), the European call option values computed by the B-S-M model are same as the H-S-V model. Consequently, the graphical representations of option price variation plotted against movements in affecting factors (say, stock price, strike price and so on) generated by these models fall over each other uniformly. Moreover, the option sensitivities (i.e. Greeks) calculated for both these models under this particular environment are identical and the corresponding diagrams drawn against varying stock prices overlap. On the other hand, for different settings of $\theta$ and $\sigma$ (Data Set 2), we notice that the option values executed by both these models differ slightly though the effect of changes in affecting factors are of similar types. In addition, the H-S-V prices display flat movements compared to B-S-M prices and this model is thus more realistic. Though the Greeks exhibit similar characteristics, the H-S-V Greeks gives different values than those of B-S-M model with the changing stock prices. Lastly, we can say that, due to the assumption of constant volatility the B-S-M option price is easier and less time consuming to calculate but sometimes it gives inaccurate results. On the contrary, the $\mathrm{H}$ $\mathrm{S}-\mathrm{V}$ model is more compatible with the real market though it takes much time to evaluate option price. It is because of the consideration of the randomness of volatility. Though we computed and observed all the outcomes of B-S-M and H-S-V models here for European call options, these models can be used to achieve similar results for other options as well.

\section{REFERENCES}

[1] John C. Hull, Options, Futures and Other Derivatives, Global edition, Eighth edition, Pearson Education Limited (2015-2016).

[2] Nicolas Privault, Stochastic Finance: An Introduction with Market Examples, CRC Financial Mathematics Series (2014).

[3] Johnson H. and Shanno D., Option Pricing when the Variance Is Changing. Journal of Finance and Quantitative Analysis, 22 (1987), 143-151.

[4] Wiggins J. B., Option Values under Stochastic Volatility: Theory and Empirical Estimates. Journal of Financial Economics, 19 (1987), 351-372.

[5] Hull J. and White a., The Pricing of Options on Assets with Stochastic Volatilities. Journal of Finance, 42 (1987), 281-300.

[6] Heston S. L., A Closed-Form Solution for Options with Stochastic Volatility, with Application to Bond and Currency Options. Review of Financial Studies, 6 (1993), 327-343.

[7] Mitun Kumar Mondal, Md. Abdul Alim, Md. Faizur Rahman, Md. Haider Ali Biswas, Mathematical Analysis of Financial Model on Market Price with Stochastic Volatility. 
[8] Scott L. O., Option Pricing When the Variance Changes Randomly: Theory, Estimation and an Application. Journal of Financial and Quantitative Analysis, 22 (1987), 419-438.

[9] Fabrice Douglas Rouah, The Heston Model and Its Extensions in Matlab and C++, John Wiley and Sons, Inc., Hoboken, New Jersey (2013).

[10] Ser-Huang Poon, A Practical Guide to Forecasting Financial Market Volatility, John Wiley and Sons limited (2005).

[11] Yuh-Dauh Lyuu, FINANCIAL ENGINEERING AND COMPUTATION: Principles, Mathematics, Algorithms. Cambridge University Press, United Kingdom (2002).

[12] M. Nurul Islam, An Introduction to Statistics and Probability, Book World (2004).

[13] Hull J. and White a., An Analysis of the Bias in Option Pricing Caused by a Stochastic Volatility. Advances in Futures and Options Research, 3 (1988), 27-61.

[14] Black, Fischer and Myron Scholes, The Pricing of Options and Corporate Liabilities, Journal of Political Economy 81.3 (1973).

[15] Yuan Yang, Valuing a European Option with the Heston Model, Rochester Institute of Technology (2013).

[16] Gurdip Bakshi, Charles Cao and Zhiwu Chen, Empirical Performance of Alternative Option Pricing Models, The Journal of THE AMERICAN FINANCE ASSOCIATION (1997). 